\title{
Intestinal lymphangiectasia - a report of two cases
}

\author{
Rashmi M. V. • Niranjana Murthy B. $\cdot$ Hephzibah Rani $\cdot$ Kodandaswamy C. R. $・$ Srinivas Arava
}

Received: 13 June 2008 / Accepted: 25 September 2008

(C) Association of Surgeons of India 2010

\begin{abstract}
Intestinal lymphangiectasia is a rare benign disease characterised by focal or diffuse dilation of the mucosal, submucosal and subserosal lymphatics. Clinically, the patients may present with protein losing enteropathy (PLE), hypoproteinemic oedema, chylous ascites, pleural effusion, acute appendicitis and intestinal obstruction.
\end{abstract}

Keywords Intestinal lymphangiectasia $\cdot$ Acute intestinal obstruction $\cdot$ Polypoidal mass $\cdot$ Acute appendicitis

Rashmi M. V. ${ }^{1}$ Niranjana Murthy B. ${ }^{1} \cdot$ Hephzibah Rani ${ }^{1}$.

Kodandaswamy C. R. ${ }^{1} \cdot$ S. Arava ${ }^{2}$

${ }^{1}$ Department of Pathology,

${ }^{2}$ Department. of Surgery,

Sree Siddhartha Medical College and Research Centre,

Tumkur - 572107, Karnataka, India

Rashmi M. V. $(\bowtie)$

E-mail: rashmiarava@yahoo.com

\section{Introduction}

Two cases of localised intestinal lymphangiectasia are being reported for there rarity of involving the small intestine, regional lymph nodes and appendix and even rarer presentation as polypoidal mass causing obstruction.

\section{Clinical summary}

Case 1: A 35-year-old malnourished female presented with colicky abdominal pain and vomiting for 2 days. Patient had no significant past history. Abdominal examination revealed guarding and tenderness all over the abdomen with absent bowel sounds. Straight X-ray abdomen revealed multiple fluid levels. A preoperative diagnosis of acute intestinal obstruction was considered, and resection of a segment of the small intestine was done with end-to-end anastomosis.

Case 2: A 21-year-old male presented with pain abdomen. On examination, right iliac fossa tenderness was noticed and acute appendicitis was suspected and ultrasound confirmed the diagnosis. Appendicectomy was performed.

\section{Pathological findings}

Case 1: On gross examination, the small intestine measured $12 \mathrm{~cm}$. Cut section of the resected bowel showed a polypoidal mass (Fig. 1) firm in consistency measuring $1.8 \times 1 \times 0.5$ $\mathrm{cm}$. Cut section of the polypoidal mass was yellowish white. A single mesenteric lymph node measuring $1 \times 0.5$ $\mathrm{cm}$ was isolated. Light microscopic examination from the intestine revealed multiple dilated lymphatic spaces lined by flattened endothelium in the mucosa and submucosa along with distorted villi (Fig. 2).

Lymph node also showed dilated lymphatics. 


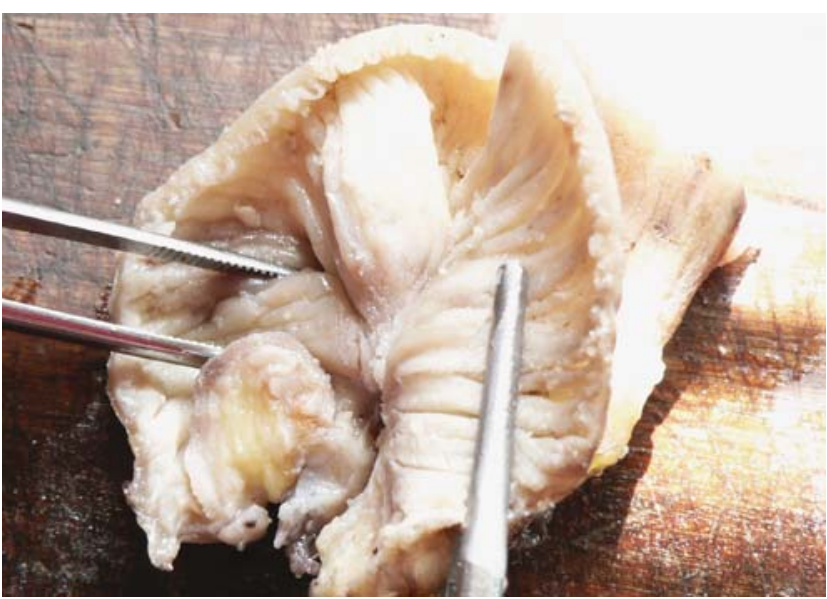

Fig. 1 Gross photograph showing resected segment of bowel with polypoidal mass

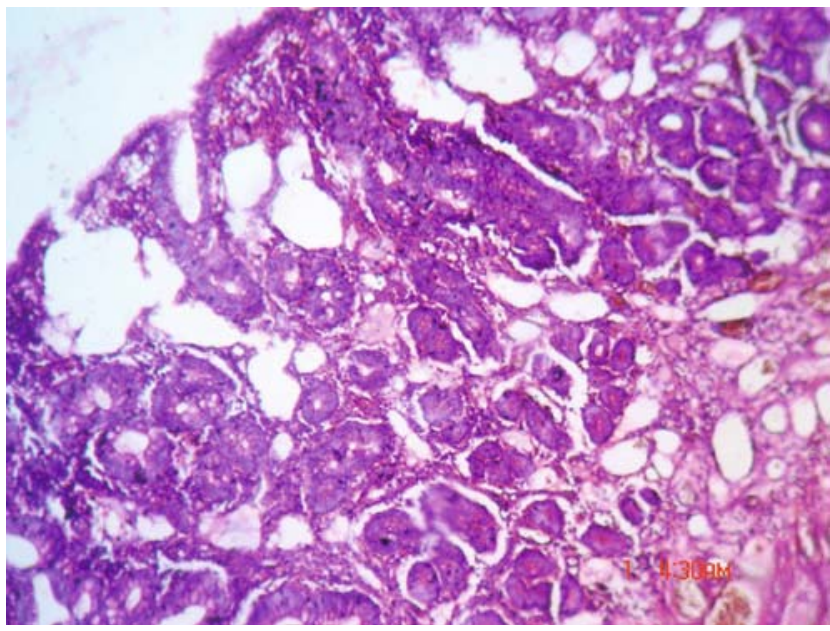

Fig. 2 Photomicrograph of the small intestine showing dilated lymphatic spaces in the mucosa and submucosa along with distorted villi $(\mathrm{H} \& \mathrm{E}, \times 100)$

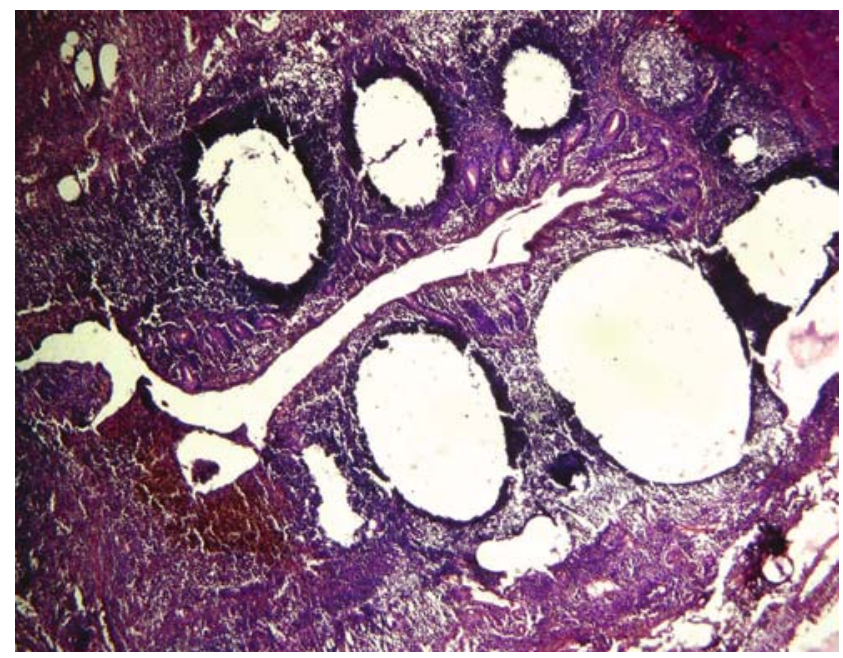

Fig. 3 Photomicrograph of the appendix showing dilated lymphatic spaces lined by flattened endothelium in the mucosa, submucosa and within the germinal follicles $(H \& E, \times 100)$
Case 2: Appendicectomy specimen measured $6 \mathrm{~cm}$. External surface was congested. Cut section showed obliteration of the lumen. Histopathological sections showed focal ulceration of the mucosa, neutrophilic infiltrate in the muscularis propria, lymphoid follicular hyperplasia and dilated lymphatic spaces lined by flattened endothelium in the mucosa, submucosa and within the follicles (Fig. 3).

\section{Discussion}

Intestinal lymphangiectasia is a rare disease which can occur in two forms - primary and secondary. Primary forms have a predilection for children and it is caused by a congenital obstructive defect of the lymphatics [1]. Congenital defects of the lymphatics constitutes a spectrum of disorders that may manifest with a variety of clinical presentations including lymphoedema, chylous effusions, lymphangiomatous malformations with cystic masses, localised gigantism and intestinal lymphangiectasia with malabsorption. These entities constitutes a relatively rare group of disorders, the origin of which remains somewhat controversial, but in some it appears to be due to early lymphatic obstruction [2]. Secondary forms are associated with many diseases, including retroperitoneal fibrosis, pancreatitis, constrictive pericarditis, primary myocardial disease, trauma, intestinal Behcet's disease, intestinal malignancy, sarcoidosis, postradiation, postsurgical and post-Fontan procedure for tricuspid atresia $[1,3]$. Intestinal lymphangiectasia may be generalised or localised, depending on the site of blockage of the mesenteric lymphatic drainage [4].

The primary form presents by 30 years of age with equal predilection in both the sexes. Oedema, hypoproteinemia, diarrhoea and lymphocytopenia are seen. The gastrointestinal symptoms are variable from mild symptoms to very severe presentation [5]. Occasionally, the patient may present with intestinal obstruction as in our case.

The severe degree of malabsorption seen in some patients is due to severe and diffuse involvement of the entire length of the small intestine. This can be associated with chylous effusions [6].

The commonest sites of occurrence of lymphangiectasia are head, neck and axilla with few cases occurring in parenchymal organs. Involvement of abdominal organs is quite rare and constitutes $1.6 \%$ of all the cases and most common intra-abdominal location is the mesentry followed by the omentum, mesocolon, retroperitoneum. Small intestinal and appendiceal involvement are extremely rare [7].

Histopathology is diagnostic and hallmarked by the presence of dilated lymphatics in the mucosa and submucosa with distension of the villi. The histopathology is identical in both the primary and secondary intestinal lymphangiectasias. Similar changes can be seen in the regional lymph nodes [1]. 
Treatment includes correction of the underlying condition and dietary manipulation. In some localised forms of intestinal lymphangiectasia, resection is the treatment as in our cases [1].

\section{Conclusion}

Primary intestinal lymphangiectasia can present rarely as a polypoidal mass causing acute intestinal obstruction. Small intestine, regional lymph nodes and appendix, being rare sites of involvement by lymphangiectasia.

\section{References}

1. Robert EP (2004) Non neoplastic intestinal diseases In: Sternberg's Diagnostic Surgical Pathology, 4th edition,
Lippincott Williams and Wilkins, Philadelphia 2:1475-1841

2. Levine C (1989) Primary disorders of the lymphatic vessels a unified concept. J Pediatr Surg 24(3):233-240

3. Conner FL, Angelides S, Gibson M, et al. (2003) Successful resection of localized intestinal lymphangiectasia postFontan: role of $(99 \mathrm{~m})$ technetium - dextran scintigraphy. Pediatrics 112:e242-247

4. Yang DM, Jung DH (2003) Localized intestinal lymphangiectasia: CT findings. Am J Roent 180:213-214

5. Case 7 (1999) A 50-year-old women with severe diarrhea during treatment for resected metastatic melanoma. New Eng J Med 340(10):789-796

6. Mistilis SP, Skyring AP, Stephen DD (1965) Intestinal lymphangiectasia, mechanism of enteric loss of plasma protein and fat. Lancet 1:77-80

7. Kaushik R, Kaushik SL, Sharma J, Sharma BB (1999) Lymphangiectasia of small intestine. Indian Pediatrics 36: 617-619 